\title{
Erratum - Some Characterizations of Mixed Poisson Processes
}

\author{
D.P. Lyberopoulos and N.D. Macheras \\ University of Piraeus, Piraeus, Greece
}

$\begin{aligned} \text { Erratum to: } & \text { Sankhyā 74-A, Part 1, pp. 57-79 } \\ & \text { (doi:10.1007/s13171-012-0011-y) }\end{aligned}$

The original paper cited above has a gap in the proofs of Lemmas 4.1 and 4.6 (and hence also in Corollary 4.2 and Proposition 4.8, which use these two lemmas). The following additional assumption needs to be inserted in the above results. Since this assumption is satisfied by any claim number process, our main results remain valid.

Assumption A. Let $X:=\left\{X_{t}\right\}_{t \in \mathbf{R}_{+}}$be a process with right-continuous paths such that its canonical filtration $\mathcal{F}:=\left\{\mathcal{F}_{t}\right\}_{t \in \mathbf{R}_{+}}$is also right-continuous.

Sketch OF THE PROOF OF Lemma 4.1. For the "only if" part, assuming that $X$ is $P$-conditionally independent, it follows that there exists a $P_{\Theta^{-n u l l ~ s e t ~}} N \in \mathfrak{B}$ such that, for any $\theta \notin N,\left\{X_{t}\right\}_{t \in \mathbf{Q}_{+}}$is $P_{\theta^{-}}$ independent, i.e., the condition $(*): \mathbf{E}_{P_{\theta}}\left[\chi_{A} f\left(X_{t}\right)\right]=P_{\theta}(A) \mathbf{E}_{P_{\theta}}\left[f\left(X_{t}\right)\right]$ is true, for all $s, t \in \mathbf{Q}_{+}$with $s<t$, all bounded Borel measurable $f: \mathbf{R} \rightarrow \mathbf{R}$ and all $A \in \mathcal{F}_{s}$. Using standard arguments, condition $(*)$ can be proved for any $s, t \in \mathbf{R}_{+}$with $s<t$.

In Lemma 4.6, put $\widetilde{\mathcal{F}}:=\left\{\widetilde{\mathcal{F}}_{t}\right\}_{t \in \mathbf{R}_{+}}$, assume for $(i) \Longrightarrow(i i)$ as well as for Proposition 4.8, that $X$ is non-decreasing, and insert assertion "In particular, if $\Theta \in \mathcal{L}^{1}(P)$ then $\left\{N_{t}-t \Theta\right\}_{t \in \mathbf{R}_{+}}$is a $(P, \widetilde{\mathcal{A}})$-martingale if and only if $\left\{N_{t}-t \theta\right\}_{t \in \mathbf{R}_{+}}$is a $\left(P_{\theta}, \widetilde{\mathcal{A}}\right)$-martingale for $P_{\Theta^{-}}$-a.a. $\theta \in \mathbf{R}$ ". In Theorem 4.10, the families $\left\{N_{t}-\mathbf{E}_{P_{\theta}}\left[N_{t}\right]\right\}_{t \in \mathbf{R}_{+}}$and $\left\{N_{t}-\mathbf{E}_{P}\left[N_{t} \mid \Theta\right]\right\}_{t \in \mathbf{R}_{+}}$must be replaced by $\left\{N_{t}-t \theta\right\}_{t \in \mathbf{R}_{+}}$and $\left\{N_{t}-t \Theta\right\}_{t \in \mathbf{R}_{+}}$respectively, with the proof remaining unchanged.

\section{D.P. Lyberopoulos and N.D. MaCheras}

Department of Statistics and Insurance Science

University of Piraeus, 80 Karaoli and Dimitriou street

18534 Piraeus, Greece

E-mail: dilyber@webmail.unipi.gr

macheras@unipi.gr 